\title{
Ultra-wideband local positioning for smart home applications
}

\begin{abstract}
Due to the inherently fine temporal resolution of UWB, arriving multi-path components can be sharply timed at a receiver to provide accurate time of arrival estimates. This characteristic makes UWB ideal for high precision radiolocation applications. This paper focuses on feature UWB technology that makes it attractive for location precise. A Time-of-Arrival (TOA) based ranging scheme using UWB radio link is proposed. The problem of ToA estimation in multi path channels and sources of estimation error are discussed. Simulation results based on channel measurement data in indoor environment are presented. Results show that the accuracy enhancement depends on two principal factors: the strength of multipath components off and the variance of non-line-of-sight (NLOS) delays (dü). For network synchronization, initial acquisition scheme for UWB multi path environments are investigated.
\end{abstract}

Keyword: NLOS; Smart home; Time acquisition; TOA; Ultra-wideband 